\title{
Excitonic Properties of Ordered Metal Nanocluster Arrays: 2D Silver Clusters at Multiporphyrin Templates
}

\author{
Merle I. S. Röhr, Polina G. Lisinetskaya, Roland Mitric* \\ Institut für physikalische und theoretische Chemie, Julius-Maximilians-Universität Würzburg, \\ Emil-Fischer-Strasse 42, D-97074 Würzburg, Germany \\ E-mail: roland.mitric@uni-wuerzburg.de
}

${ }^{*}$ To whom correspondence should be addressed 


\begin{abstract}
The design of ordered arrays of metal nanoclusters such as for example 2D cluster organic frameworks might open a new route towards the development of materials with tailored optical properties. Such systems could serve as plasmonically enhanced light-harvesting materials, sensors or catalysts. We present here a theoretical approach for the simulation of the optical properties of ordered arrays of metal clusters that is based on the ab initio parametrized Frenkel exciton model. We demonstrate that small atomically precise silver clusters can be assembled in one- and two-dimensional arrays on suitably designed porphyrin templates exhibiting remarkable optical properties. By employing explicit TDDFT calculations on smaller homologs, we show that the intrinsic optical properties of metal clusters are largely preserved but undergo J- and H-type excitonic coupling that results in controllable splitting of their excited states. Furthermore, ab initio parameterized Frenkel exciton model calculations allow us to predict an energetic splitting of up to $0.77 \mathrm{eV}$ in extended two-dimensional square arrays and $0.79 \mathrm{eV}$ in tilted square aggregates containing up to 25 cluster-porphyrin subunits.
\end{abstract}

\title{
Introduction
}

The design of multi-chromophoric molecular aggregates with tailored optical properties continues to be an area of strong interest since these systems might find numerous applications as superior light-harvesters in solar cells or in novel optoelectronic devices. ${ }^{1,2}$ The distinct changes in the absorption spectra of these assemblies compared to the monomeric species can be attributed to electronic couplings between the constituents. This leads to the energetic splitting of the absorption bands as well as to the redistribution of their intensities, allowing to optimally tune optical properties of such aggregates. ${ }^{3}$ In the frame of the Frenkel exciton model, the spectral features can be explained by coupling of the transition dipole moments between the chromophores where in extreme cases the head-to-tail orientation leads to J-type coupling with red-shifted absorption, while a parallel orientation leads to H-type coupling exhibiting blue-shifted absorption. ${ }^{4}$

While molecular dye aggregates have been widely studied, little is known about the optical 
properties of self-organized metal nanoclusters in a size regime where "each atom counts" $(\sim 1$ $2 \mathrm{~nm})$. The usage of small metal clusters as chromophores in such assemblies would open a route towards an efficient "tuning" of the absorption bands in the desired energetic range since cluster excitation energies are strongly size and structure dependent. ${ }^{5,6}$ Furthermore, clusters can exhibit bright fluorescence as well as intense linear and even nonlinear absorption and thus have optical properties which are in many respects superior to those of organic dyes. ${ }^{7,8}$ We have shown recently that Ag cluster arrays can serve as efficient systems for light-induced exciton transport due to a strong electronic coupling between individual "magic" $\mathrm{Ag}_{8}$ clusters. ${ }^{9,10}$ In addition, Ag clusters exhibit a high affinity to undergo covalent binding to biomolecules, which makes them ideal candidates for the preparation of cluster-organic aggregates. ${ }^{11-13}$ As an example, DNA origami scaffolds have been used in order to assemble metal nanoclusters in a precisely defined threedimensional architecture. ${ }^{14,15}$ Various organic ligands can help to stabilize clusters as it has been shown in several recent studies. ${ }^{16,17}$ In particular, quantum sized nanoparticles can plasmonically enhance the optical properties of attached biomolecules and organic dyes. ${ }^{18}$ An attractive possibility to develop novel optical cluster-based materials is the self-organization at two-dimensional templates with well-defined order, comparable to the known 2D metal-organic frameworks (2D MOF). First steps in this direction have been recently made by the preparation of a novel surface coordination network based on $\mathrm{Cu}_{3}$ nanoclusters connected via tetrahydrobenzene (THB). ${ }^{19}$ Only recently, experimentalists have also shown for the first time the dominant role of dipole-dipole interaction inside a two-dimensional molecular aggregate (2DMA) placed on a metallic substrate, in determining its ultrafast quenching and energy-dissipation rate. ${ }^{20}$

In this article, we introduce a new 2D cluster organic framework consisting of "magic" $\mathrm{Ag}_{3}^{+}$ clusters bound to triply fused porphyrin arrays. Porphyrins have previously served as organic linkers in the preparation of surface assisted metal-organic $2 \mathrm{D}$ architectures. ${ }^{21}$ Also, synthesis of 1D porphyrin tapes and small sheets has been reported ${ }^{22,23}$ and surface-assisted preparation of porphyrin-oligomers has been demonstrated on an Ag (111) support. ${ }^{24}$

Here, we explore systematically the coupling between the Ag clusters at porphyrin templates and address the optical properties of such arrays, which might find applications in light-harvesting, 

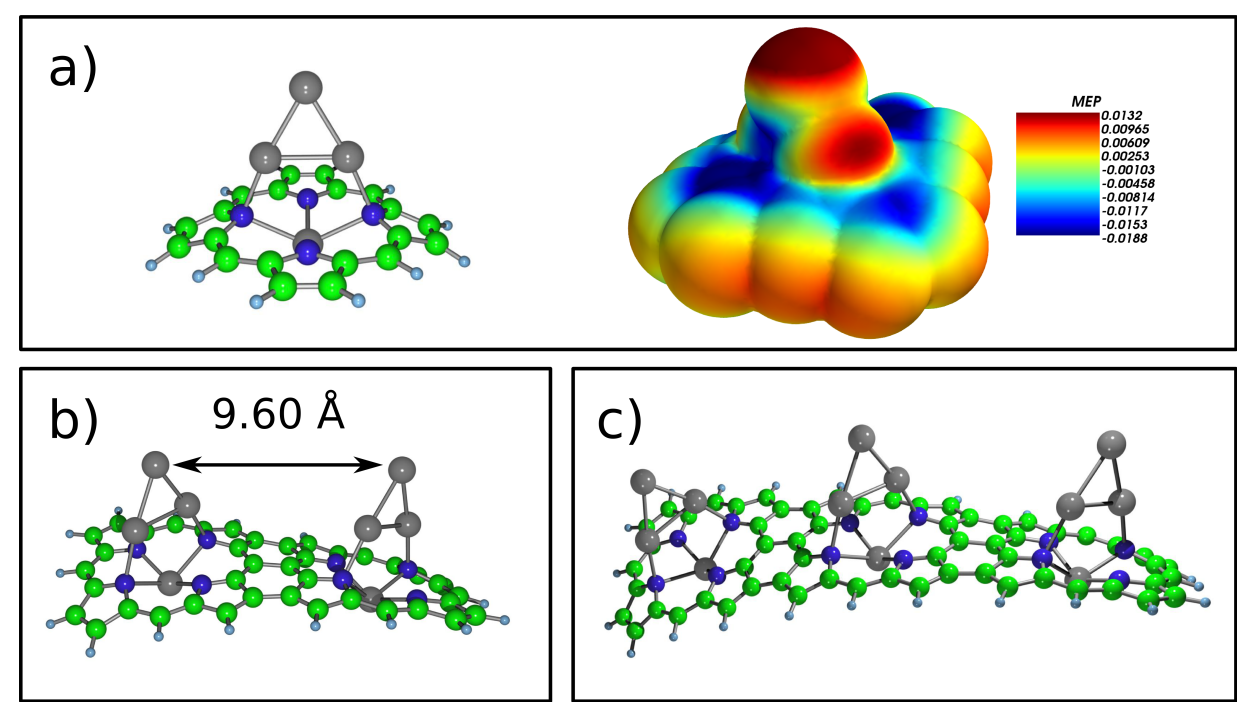

Figure 1: DFT optimized structures for a) monomeric cluster-porphyrin hybrid system, b) dimer and c) linear trimer. The electrostatic potential map is shown in a), illustrating a positive charge located at the silver cluster.

analytical sensing or cluster-mediated catalysis. ${ }^{25,26}$

\section{Methods}

The extensive search for structures was performed by the simulated annealing procedure coupled with molecular dynamics simulations in the framework of the RI-DFT method ${ }^{27}$ and the BLYP functional. ${ }^{28}$ The structures found were re-optimized using the CAM-B3LYP functional ${ }^{29}$ with a 19-electron relativistic effective core potential ${ }^{30}$ (19el-RECP) for silver. For the porphyrin moiety, the def2-TZVP basis set was used. ${ }^{31}$ The absorption spectra were obtained in the framework of TDDFT using the 11el-RECP with the corresponding basis set which has been developed for an accurate description of excited states of silver clusters. ${ }^{32}$ The absorption spectra of larger arrays have been obtained by employing the transition density cube (TDC) method. ${ }^{33}$

\section{Simulation of cluster-array spectra}

We assume that an array under consideration consists of $N$ weakly interacting metal clusters. The total electronic Hamiltonian of the cluster array is constructed based on the Hamiltonians of indi- 
vidual components as

$$
\hat{\mathrm{H}}=\sum_{I=1}^{N} \hat{\mathrm{H}}_{I}^{0}+\sum_{I=1}^{N} \sum_{J>I} \hat{\mathrm{V}}_{I J}
$$

where $\hat{\mathrm{H}}_{I}^{0}$ is the electronic Hamiltonian of the $I$-th cluster, which can be constructed based on the TDDFT calculations, and $\hat{\mathrm{V}}_{I J}$ is the interaction between the $I$-th and $J$-th clusters, which within the described approach is considered to be purely electromagnetic and can be naturally divided into the electron-electron, electron-nuclear and nuclear-nuclear contributions:

$$
\hat{\mathrm{V}}_{I J}=\sum_{p q} \frac{1}{\left|\mathbf{r}_{p}^{I}-\mathbf{r}_{q}^{J}\right|}-\sum_{p B} \frac{Q_{B}}{\left|\mathbf{r}_{p}^{I}-\mathbf{r}_{B}^{J}\right|}-\sum_{q A} \frac{Q_{A}}{\left|\mathbf{r}_{A}^{I}-\mathbf{r}_{q}^{J}\right|}+\sum_{A B} \frac{Q_{A} Q_{B}}{\left|\mathbf{r}_{A}^{I}-\mathbf{r}_{B}^{J}\right|}=\hat{\mathrm{V}}_{I J}^{e e}+\hat{\mathrm{V}}_{I J}^{e n}+\mathrm{V}_{I J}^{n n}
$$

Here indices $p, q$ run over all electrons and $A, B$ over all nuclei of the $I$-th and $J$-th clusters, respectively.

The natural basis for the representation of the coupled array Hamiltonian $\hat{\mathrm{H}}$ is the product-state basis built up from the individual cluster eigenfunctions:

$$
\left|\phi_{i j \ldots z}\right\rangle=\left|\Psi_{i}^{1}\right\rangle \otimes\left|\Psi_{j}^{2}\right\rangle \otimes \ldots \otimes\left|\Psi_{z}^{N}\right\rangle
$$

where each eigenfunction $\left|\Psi_{i}^{I}\right\rangle$ satisfies the time-independent Schrödinger equation $\hat{\mathrm{H}}_{I}^{0}\left|\Psi_{i}^{I}\right\rangle=$ $E_{i}^{I}\left|\Psi_{i}^{I}\right\rangle$ with $E_{i}^{I}$ being the $i$-th electronic state energy of the $I$-th cluster, and the indices $i, j, \ldots, z$ running over all included electronic states for each cluster in the array. In this basis the Hamiltonian $\hat{\mathrm{H}}$ can be represented by a super-matrix:

$$
\left.(\hat{\mathrm{H}})_{i j \ldots z, i^{\prime} j^{\prime} \ldots z^{\prime}}=\sum_{I=1}^{N}\left\langle\phi_{i j \ldots z}\left|\hat{\mathrm{H}}_{I}^{0}\left(\mathbf{r}_{p}^{I}\right)\right| \phi_{i^{\prime} j^{\prime} \ldots z^{\prime}}\right\rangle+\sum_{I=1 J>I}^{N} \sum_{\phi_{i j \ldots z}}\left|\hat{\mathrm{V}}_{I J}\left(\mathbf{r}_{p}^{I}, \mathbf{r}_{q}^{J}\right)\right| \phi_{i^{\prime} j^{\prime} \ldots z^{\prime}}\right\rangle
$$

The first term in Eq. (4) in the basis (3) is a diagonal matrix due to the orthogonality of the single cluster eigenfunctions $\left\langle\Psi_{i}^{I} \mid \Psi_{i^{\prime}}^{J}\right\rangle=\delta_{i i^{\prime}}$ with the elements 


$$
\sum_{I=1}^{N}\left\langle\phi_{i j \ldots z}\left|\hat{\mathrm{H}}_{0}^{I}\left(\mathbf{r}_{p}^{I}\right)\right| \phi_{i j \ldots z}\right\rangle=E_{i}^{1}+E_{j}^{2}+\cdots+E_{z}^{N}
$$

At the same time, the second term in (4) is represented by a non-diagonal matrix

$$
\begin{aligned}
& \left.\sum_{I=1 J>I}^{N} \sum_{J j \ldots z}\left|\hat{\mathrm{V}}_{I J}\left(\mathbf{r}_{p}^{I}, \mathbf{r}_{q}^{J}\right)\right| \phi_{i^{\prime} j^{\prime} \ldots z^{\prime}}\right\rangle= \\
& \sum_{I=1 J>I}^{N} \sum_{k}\left[\left\langle\Psi_{k}^{I}\left|\otimes\left\langle\Psi_{l}^{J}\left|\hat{\mathrm{V}}_{I J}^{e e}\left(\mathbf{r}_{p}^{I}, \mathbf{r}_{q}^{J}\right)\right| \Psi_{k^{\prime}}^{I}\right\rangle \otimes\right| \Psi_{l^{\prime}}^{J}\right\rangle+\right. \\
& \left.\left\langle\Psi_{k}^{I}\left|\hat{\mathrm{V}}_{I J}^{e n}\left(\mathbf{r}_{p}^{I}\right)\right| \Psi_{k^{\prime}}^{I}\right\rangle \delta_{l l^{\prime}}+\left\langle\Psi_{l}^{J}\left|\hat{\mathrm{V}}_{I J}^{e n}\left(\mathbf{r}_{q}^{J}\right)\right| \Psi_{l^{\prime}}^{J}\right\rangle \delta_{k k^{\prime}}+\mathrm{V}_{I J}^{n n} \delta_{k k^{\prime}} \delta_{l l^{\prime}}\right] \delta_{i i^{\prime}} \delta_{j j^{\prime}} \ldots \delta_{z z^{\prime}}
\end{aligned}
$$

with the $I$-th and $J$-th cluster indices omitted in the product $\delta_{i i^{\prime}} \delta_{j j^{\prime}} \ldots \delta_{z z^{\prime}}$. Using Eq. (2) we reduce the electron-electron interaction element in Eq. (6) to the following integral

$$
\begin{aligned}
&\left\langle\Psi_{k}^{I}\left|\otimes\left\langle\Psi_{l}^{J}\left|\sum_{p q} \frac{1}{\left|\mathbf{r}_{p}^{I}-\mathbf{r}_{q}^{J}\right|}\right| \Psi_{k^{\prime}}^{I}\right\rangle \otimes\right| \Psi_{l^{\prime}}^{J}\right\rangle= \\
& N_{I} N_{J} \int \ldots \int \Psi_{k}^{I *}\left(\mathbf{x}_{I}\right) \Psi_{l}^{J *}\left(\mathbf{x}_{J}\right) \frac{1}{\left|\mathbf{r}_{1}^{I}-\mathbf{r}_{1}^{J}\right|} \Psi_{k^{\prime}}^{I}\left(\mathbf{x}_{I}\right) \Psi_{l^{\prime}}^{J}\left(\mathbf{x}_{J}\right) \mathrm{d} \mathbf{x}_{I} \mathrm{~d} \mathbf{x}_{J} .
\end{aligned}
$$

Here $\mathbf{x}_{I, J}$ denote the position and spin variables of all electrons of the $I$-th $\left(J\right.$-th) cluster, $\mathbf{r}_{1}^{I, J}$ is the position of the first electron of the cluster $I$ or $J$, and $N_{I, J}$ is the number of electrons in the clusters $I$ or $J$. Defining a transition density as

$$
\rho_{k k^{\prime}}^{I}\left(\mathbf{x}_{1}^{I}\right)=N_{I} \int \ldots \int \Psi_{k}^{I *}\left(\mathbf{x}_{I}\right) \Psi_{k^{\prime}}^{I}\left(\mathbf{x}_{I}\right) \mathrm{d} \mathbf{x}_{2}^{I} \ldots \mathrm{d} \mathbf{x}_{N_{I}}^{I}
$$

where the integration runs over position and spin variables of the $I$-th cluster electrons from the second to the $N_{I}$-th. Note, that in case $k=k^{\prime}$, Eq. (8) gives the one-electron density of the $k$-th state. Substituting Eq. (8) to Eq. (7), we derive the electron-electron interaction element between the $I$-th and $J$-th clusters based on the transition densities 


$$
\left\langle\Psi_{k}^{I}\left|\otimes\left\langle\Psi_{l}^{J}\left|\sum_{p q} \frac{1}{\left|\mathbf{r}_{p}^{I}-\mathbf{r}_{q}^{J}\right|}\right| \Psi_{k^{\prime}}^{I}\right\rangle \otimes\right| \Psi_{l^{\prime}}^{J}\right\rangle=\iint \frac{\rho_{k k^{\prime}}^{I}\left(\mathbf{r}_{1}^{I}\right) \rho_{l l^{\prime}}^{J}\left(\mathbf{r}_{1}^{J}\right)}{\left|\mathbf{r}_{1}^{I}-\mathbf{r}_{1}^{J}\right|} \mathrm{d} \mathbf{r}_{1}^{I} \mathrm{~d} \mathbf{r}_{1}^{J} .
$$

In the same manner the electron-nuclear interaction elements can be obtained:

$$
\left\langle\Psi_{k}^{I}\left|\sum_{p B} \frac{Q_{B}}{\left|\mathbf{r}_{p}^{I}-\mathbf{r}_{B}^{J}\right|}\right| \Psi_{k^{\prime}}^{I}\right\rangle=\sum_{B} Q_{B} \int \frac{\rho_{k k^{\prime}}^{I}\left(\mathbf{r}_{1}^{I}\right)}{\left|\mathbf{r}_{1}^{I}-\mathbf{r}_{B}^{J}\right|} \mathrm{d} \mathbf{r}_{1}^{I} .
$$

Within the described model, the electron-electron interaction (9) does not include the exchange interaction between individual subunits. However, since in our simulations the distance between the clusters is kept relatively large, such interaction is unlikely to contribute to the optical properties of the array.

When the Hamiltonian $\hat{\mathrm{H}}$ is constructed, one can diagonalize it to obtain the set of states with eigenenergies $\varepsilon_{m}$ and eigenfunctions $\left|\psi_{m}\right\rangle$ which in the tensor basis (3) are expanded as:

$$
\left|\psi_{m}\right\rangle=\sum_{i j \ldots z} C_{i j \ldots z}^{m}\left|\phi_{i j \ldots z}\right\rangle=\sum_{i j \ldots z} C_{i j \ldots z}^{m}\left|\Psi_{i}^{1}\right\rangle \otimes\left|\Psi_{j}^{2}\right\rangle \otimes \ldots \otimes\left|\Psi_{z}^{N}\right\rangle
$$

Now, the array spectrum can be straightforwardly calculated with the excitation energies

$$
E_{m}=\varepsilon_{m}-\varepsilon_{0}
$$

and oscillator strengths

$$
f_{m}=\frac{2}{3} E_{m}\left|\mathbf{M}_{0 m}\right|^{2}
$$

where $\mathbf{M}_{0 m}$ is the transition dipole moment between the ground and the $m$-th excited state of the array, which can be written in terms of the single cluster transition dipole moments $\mu_{k k^{\prime}}^{I}$ :

$$
\mathbf{M}_{0 m}=\left\langle\psi_{0}\left|\sum_{I} \sum_{p=1}^{N_{I}} \mathbf{r}_{p}^{I}\right| \psi_{m}\right\rangle=\sum_{I} \sum_{i j \ldots z} \sum_{k^{\prime}} C_{i j \ldots k \ldots z}^{* 0} C_{i j \ldots k^{\prime} \ldots z}^{m} \mu_{k k^{\prime}}^{I} .
$$

Here the indices $k$ and $k^{\prime}$ correspond to the $I$-th cluster index in the set $i j \ldots z$. 
It is now evident that the prerequisites needed to simulate the absorption spectrum of the cluster array are the electronic state energies of each single cluster (see Eq. (5)), transition densities between all the electronic states included in the simulation, and transition dipole moments between all the states. In principle, for molecular-sized subunits, any ab initio or semiempirical electronic structure method can be used to obtain these quantities. In the current contribution we have employed the linear response time-dependent density functional theory (TDDFT) due to its efficiency and applicability to relatively large complex systems. Standard TDDFT routines allow for obtaining the required excited state electronic energies as well as transition densities and transition dipole moments between the ground and excited electronic states. In contrast to the ground-excited state transitions, the determination of the excited-excited state transition densities and dipole moments requires an approximate procedure, which has been first presented in Ref. ${ }^{34}$

This procedure is based on the Casida ansatz, ${ }^{35}$ which allows the excited state electronic wave function to be approximated by the configuration interaction singles-like expansion

$$
\left|\Psi_{k}\right\rangle=\sum_{a, r} c_{a, r}^{k}\left|\Phi_{a, r}^{C S F}\right\rangle
$$

where $\left|\Phi_{i, a}^{C S F}\right\rangle$ is a singlet spin-adapted configuration state function (CSF) defined as:

$$
\left|\Phi_{a, r}^{C S F}\right\rangle=\frac{1}{\sqrt{2}}\left(\left|\Phi_{a \alpha}^{r \beta}\right\rangle+\left|\Phi_{a \beta}^{r \alpha}\right\rangle\right)
$$

and $\left|\Phi_{a \alpha}^{r \beta}\right\rangle$ is a Slater determinant with two unpaired electrons, one on the occupied Kohn-Sham (KS) orbital $a$ with spin $\alpha$ and another on the virtual orbital $r$ with spin $\beta$. Correspondingly, in the determinant $\left|\Phi_{a \beta}^{r \alpha}\right\rangle$ the unpaired electrons reside on the occupied KS orbital $a$ (spin $\beta$ ) and the virtual orbital $r$ (spin $\alpha$ ). The representation (15) is a practicable way of defining an excited state wave function based on linear response TDDFT. The coefficients $c_{a, r}^{k}$ in Eq. (15) can be determined on physical grounds by requiring that the wave function in Eq. (15) leads to the same density response as the one obtained by the linear response TDDFT procedure. Thus, for nonhybrid functionals without exact exchange the coefficients $c_{a, r}^{k}$ are given by: 


$$
c_{a, r}^{k}=\left(\frac{\varepsilon_{r}-\varepsilon_{a}}{\omega_{k}}\right)^{-1 / 2}\left(X_{a r}^{k}+Y_{a r}^{k}\right),
$$

where $\varepsilon_{a}$ and $\varepsilon_{r}$ represent the orbital energies of $a$-th occupied and $r$-th virtual single electron orbitals, respectively, $\omega_{k}$ is the excitation energy of the $k$ th electronic state, and $X^{k}$ and $Y^{k}$ are the solution of the TDDFT eigenvalue problem. ${ }^{35,36}$

Now substituting Eqs. (15) and (16) in (8) and calculating the integral, making use of mutual orthogonality of the MOs, one can derive the formulas for single-cluster transition densities between all the states of interest

$$
\begin{gathered}
\rho_{00}=2 \sum_{a} \phi_{a}^{*} \phi_{a} \\
\rho_{0 k}=2 \sum_{a r} c_{a, r}^{k} \phi_{a}^{*} \phi_{r} \\
\rho_{k k^{\prime}}=2\left(\delta_{k k^{\prime}} \rho_{00}+\sum_{a r s} c_{a, r}^{k *} c_{a, s}^{k^{\prime}} \phi_{r}^{*} \phi_{s}-\sum_{a b r} c_{a, r}^{k *} c_{b, r}^{k^{\prime}} \phi_{a}^{*} \phi_{b}\right)
\end{gathered}
$$

where $\{\phi\}$ are the MOs of the cluster, indices $a, b$ run over all occupied and $r, s$ over all virtual orbitals. After the transition densities have been calculated, the corresponding transition dipole moments can be straightforwardly determined

$$
\mu_{k k^{\prime}}=\int \mathbf{r} \rho_{k k^{\prime}}(\mathbf{r}) \mathrm{d} \mathbf{r}
$$

In practice, it is convenient to calculate MOs of a single subunit and thus the transition densities on a grid. In this case, the Eqs. (18)-(20) should be multiplied by the volume of a unit cell of the grid to ensure that the one-electron densities sum up to the number of electrons in the cluster. Additionally, the integrals in Eqs. (9)-(10) and (21) are substituted by sums over grid points.

We demonstrate the applicability of the presented method to simulations of absorption spectra of $\mathrm{Ag}_{3}^{+}$cluster arrays by comparing the spectra for monomer, dimer, and trimer, simulated using the 
proposed methodology, to the spectra calculated by means of the more accurate $a b$ initio correlated CC2 method. ${ }^{37}$ The CC2 model is an approximation to the coupled cluster singles and doubles (CCSD) model and provides excitation energies correct to the second order and transition moments correct to the first order at reasonable computation time. The resulting spectra are presented in Fig. 2.
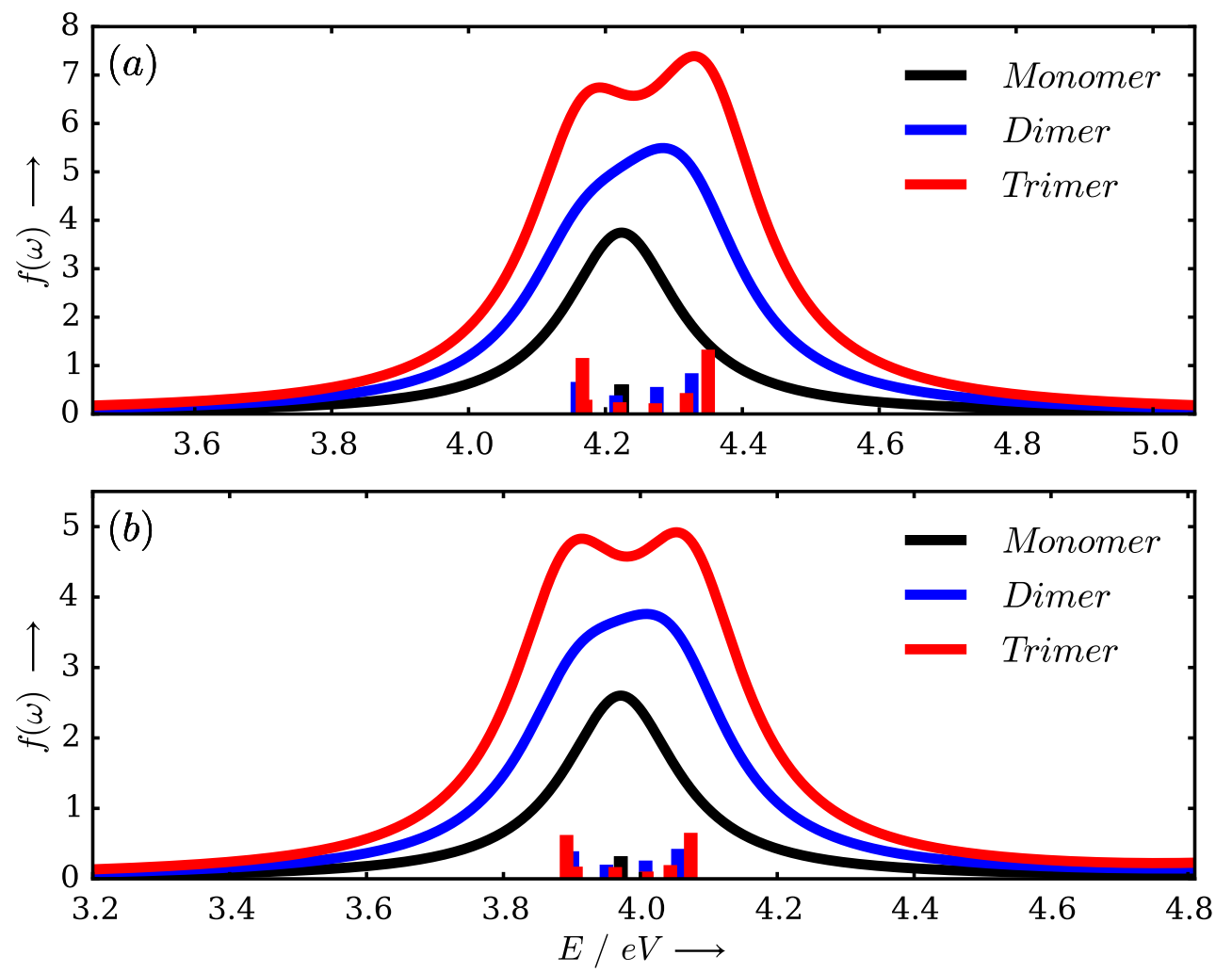

Figure 2: Absorption spectra of $\mathrm{Ag}_{3}^{+}$cluster arrays calculated using (a) the method presented in Sec. (), (b) ab initio CC2 method.

Note that the energy scales of the two plots in Fig. 2 are shifted with respect to each other by $\sim 0.25 \mathrm{eV}$ in order to locate the monomer spectra one above the other. The difference between the monomer absorption energies is due to different accuracy of the TDDFT and CC2 models. Since in our approach the array spectra are based of the monomer data, the dimer and trimer spectra demonstrate the same shift with respect to their CC2 counterparts as well. Despite this fact, all the features, such as absorption band splitting and redistribution of intensities are accurately reproduced. Thus we conclude that, for larger arrays, the absorption spectra will be reliable as 
well.

\section{Results and Discussion}

\section{Explicit Calculations}

We first present the structural and optical properties of the monomeric porphyrin- $\mathrm{Ag}_{4}$ hybrid structure in order to demonstrate the stability of such systems. The optimized ground state structure has been obtained by DFT calculations and is presented in Fig. 1. Other calculated higher energy isomers are provided in the Supporting Information (SI). The structure is composed of a triangular $\mathrm{Ag}_{3}^{+}$subunit and a distorted porphyrin moiety with two pyrrol rings folded in and additionally an "out-of-plane"-located $\mathrm{Ag}^{+}$ion. The natural bonding orbital (NBO) analysis shows a net charge of 0.77 localized at the $\mathrm{Ag}_{3}$ subunit and 0.70 for the remaining $\mathrm{Ag}^{+}$ion, thus proving the presence of a singly charged $\mathrm{Ag}_{3}^{+}$cluster, which has formally two valence electrons and a closed S-shell "magic" structure. The calculated molecular electrostatic potential map shown in Fig. 1a) further illustrates the charge distribution within the hybrid system. Experimentally, similar systems containing two bare $\mathrm{Ag}^{+}$ions are already known. ${ }^{38}$ However, in contrast to such systems the $\mathrm{Ag}_{3}^{+}$subunit still has two delocalized electrons and behaves as a stable UV chromophore as we have previously demonstrated in several species. ${ }^{18,39}$ In Fig. 3 we present the calculated absorption spectrum of the monomeric hybrid system obtained by the long-range corrected TD-DFT method. ${ }^{29}$ The spectrum exhibits four broad bands in the range between 3 and $5 \mathrm{eV}$ and an almost dark band located at $2.16 \mathrm{eV}$, which can be assigned to the characteristic porphyrin Q-band. ${ }^{40}$ The analysis of the transitions shown in the lower part of Fig. 3 reveals that the excitations marked red in the spectra can be attributed to almost pure $\mathrm{Ag}_{3}^{+}$cluster transitions. They are identified as excitations from the " $\mathrm{S}$ " cluster orbital to $\mathrm{P}_{\mathrm{x}}, \mathrm{P}_{\mathrm{y}}$, and $\mathrm{P}_{\mathrm{z}}$ cluster orbitals, respectively.

Furthermore, comparison with the calculated spectra of the bare $\mathrm{Ag}_{3}^{+}$cluster (shown in the inset, and in the SI) leads to the conclusion that the absorption properties of the cluster are largely preserved within the hybrid system, although the excited state marked "C" undergoes a shift to- 

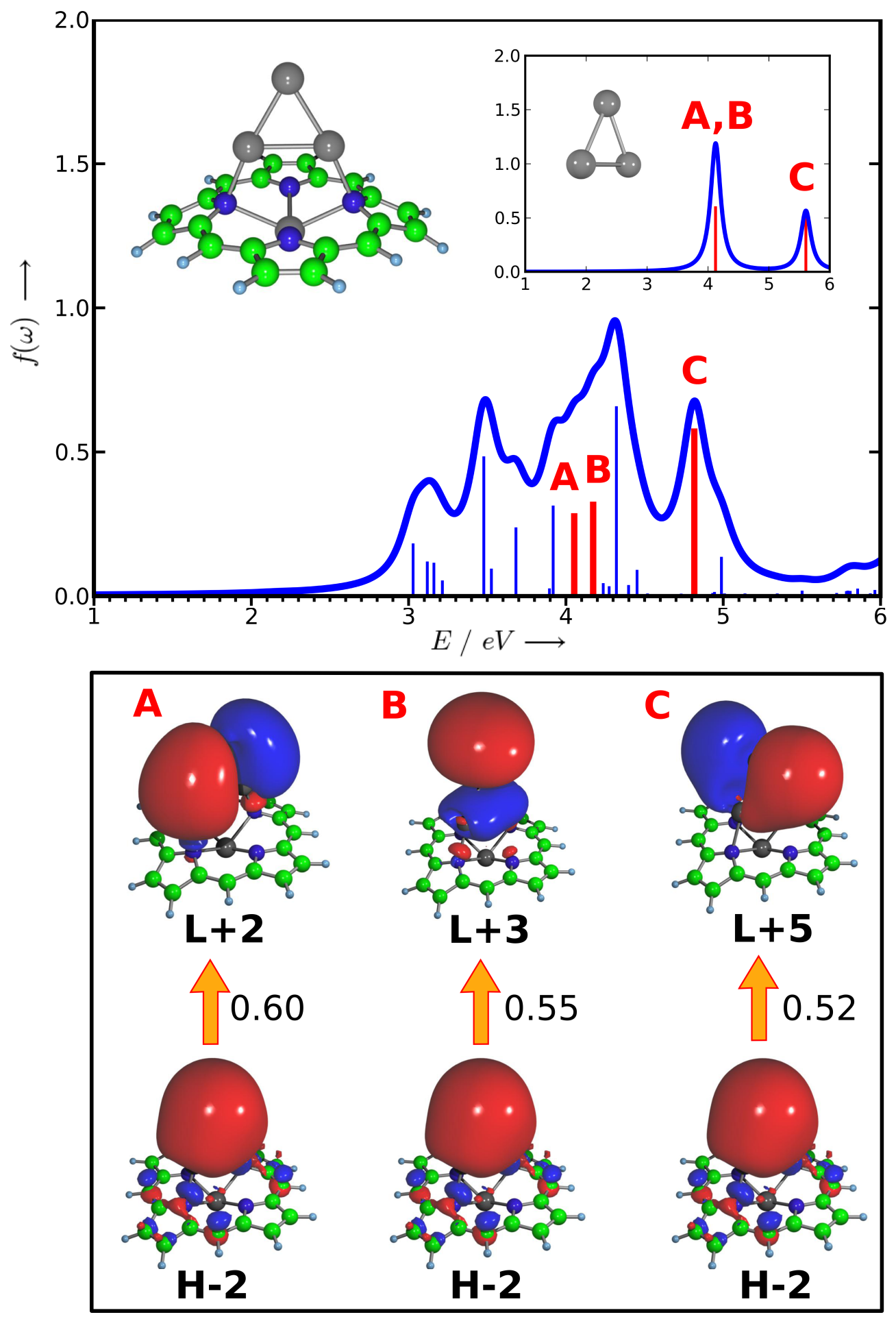

Figure 3: Top: Calculated absorption spectrum for porphyrin- $\mathrm{Ag}_{4}$. The inset shows the spectrum for the bare $\mathrm{Ag}_{3}^{+}$cluster. Bottom: Analysis of the marked excited states, only leading transitions are shown. 
wards lower energies. In order to investigate the electronic interaction between the clusters in an aggregate, we have performed explicit DFT and TDDFT calculations on the dimer and a trimer hybrid system. In these species a directly triply linked porphyrin dimer and trimer interact with $\mathrm{Ag}_{4}$ moieties. An extensive structure search shows that in both systems the most stable isomer consists of an out-of-plane $\mathrm{Ag}^{+}$ion and an $\mathrm{Ag}_{3}^{+}$cluster, similar to the monomer, whereas the clusters are oriented in a parallel direction in the dimer and trimer. The distance between the Ag atoms on top of the trimeric moieties is $9.60 \AA$ and the porphyrin backbone undergoes a twisted distortion.
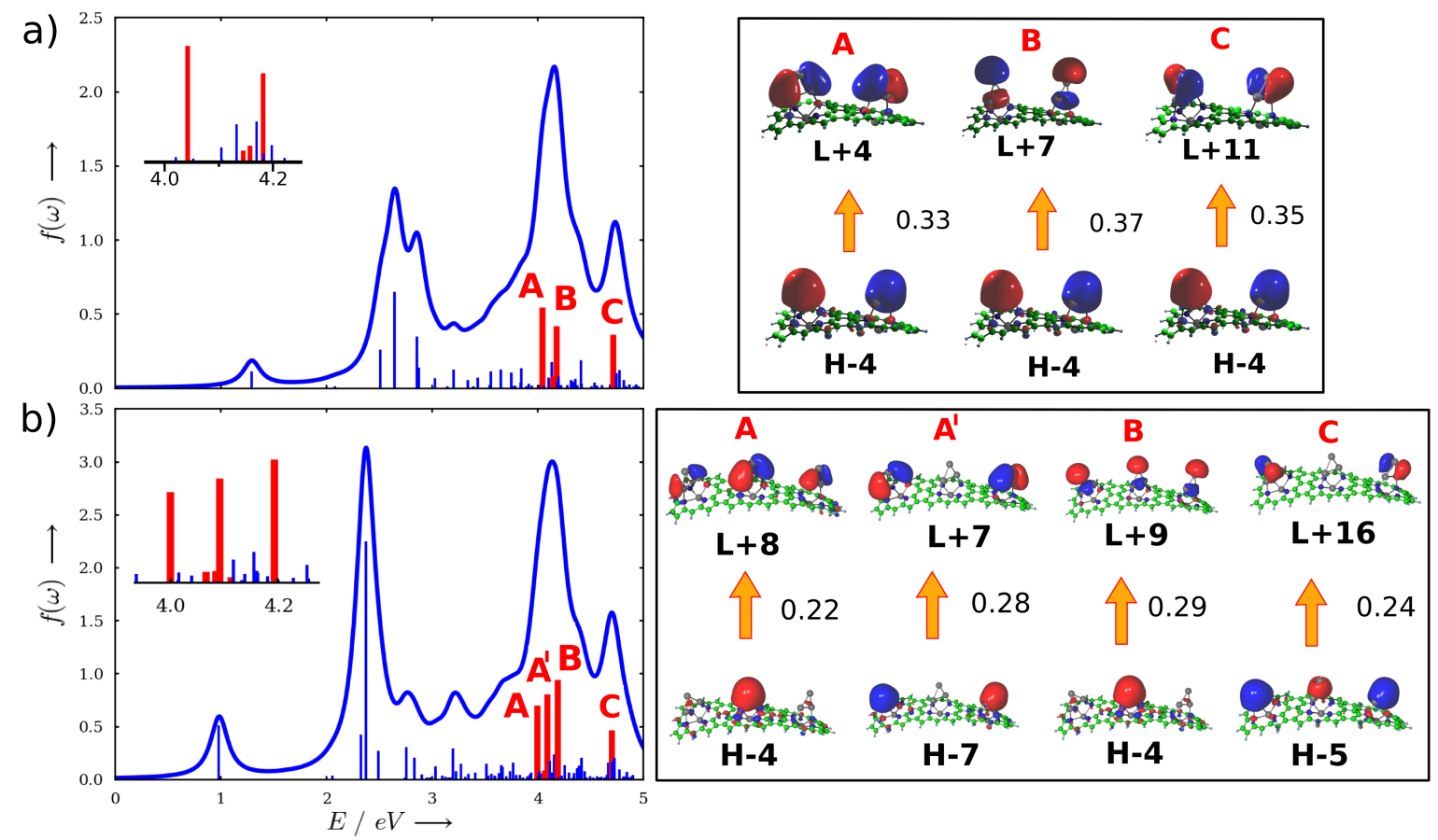

Figure 4: Calculated absorption spectra of the a) dimer and b) trimer. The analysis of the intense states is given on the right hand side.

In Fig. 4 absorption spectra of dimeric and trimeric linear hybrid tapes are presented. The spectrum of the dimer exhibits four broad bands, located between 1 and $5 \mathrm{eV}$ with a maximum at $4 \mathrm{eV}$. The spectrum of the trimer shows similar features while the first two bands are red-shifted and exhibit an increased intensity. These features are in good agreement with experimental and theoretical findings on the absorption spectra of pure porphyrin sheets and can be attributed to conjugation effects. ${ }^{41}$ Analysis of the excited states in both spectra reveals the presence of intracluster transitions at $4.0 \mathrm{eV}$ and $4.8 \mathrm{eV}$. The transitions labelled A and B can be identified as S-P 
cluster transitions, which are shifted and splitted with respect to the monomer due to excitonic coupling. In case of the dimer, the coupling results in the formation of four dimer states located at $4.04 \mathrm{eV}$ (marked "A" in Fig. 4a), $4.15 \mathrm{eV}, 4.16 \mathrm{eV}$ and $4.18 \mathrm{eV}$ (marked "B" in Fig. 4a)) resulting in a total splitting of $0.12 \mathrm{eV}$. The trimer spectrum comprises six states corresponding to the A and B transitions at $3.99 \mathrm{eV}$ (marked A in Fig 4b), $4.07 \mathrm{eV}, 4.09 \mathrm{eV}, 4.09 \mathrm{eV}$ (marked A' in Fig. 4b), $4.11 \mathrm{eV}$ and $4.19 \mathrm{eV}$ (marked B) spanning an energy range of $0.2 \mathrm{eV}$. Analysis reveals that the discussed states arise due to transitions from orbitals belonging to the cluster S-band of the polymeric system to orbitals from the $\mathrm{P}_{\mathrm{x}}$ and the $\mathrm{P}_{\mathrm{y}}$ band, and thus have the same character as the "original" states in the monomer. This pattern can be understood by examining the transition dipole moments and its orientations. As illustrated on top of Fig. 5, in case of the transition "A", the transition dipole moments are oriented in a "head to tail" manner resulting in a J-type splitting. In contrast, the orientation of the dipole moments in the "B" transition leads to H-type coupling. In case of the dimer, this leads to the formation of a less intense peak belonging to the A band at higher energy and a less intense peak belonging to the B band at lower energy (cf. Fig. 4a). The trimer exhibits three J- and three H-type states derived from the A and B bands. Notice, that the lowest $\mathrm{H}$ state belonging to the B band is even lower in energy than the highest J type state (cf. Fig. 4b). Therefore, we cannot energetically distinguish between the A and the B band anymore. The calculations show that the intrinsic optical properties of the Ag clusters are preserved in multichromophoric aggregates at conjugated porphyrin templates. Based on these findings we investigate in the following how the optical properties of the porphyrin-silver hybrid system evolve with size. Therefore, we consider a 2D arrangement of $\mathrm{Ag}_{3}^{+}$clusters with the same distances and orientation as in the dimer and trimer systems and explore the effect of electronic couplings on the strong intracluster transitions. In the first example, we assume a planar porphyrin template structure as we expect from synthesis on a support. In the second example, we consider the optical properties of extended tilted systems, based on additonal excplicit DFT calculations on the structure of such tapes and arrays. 


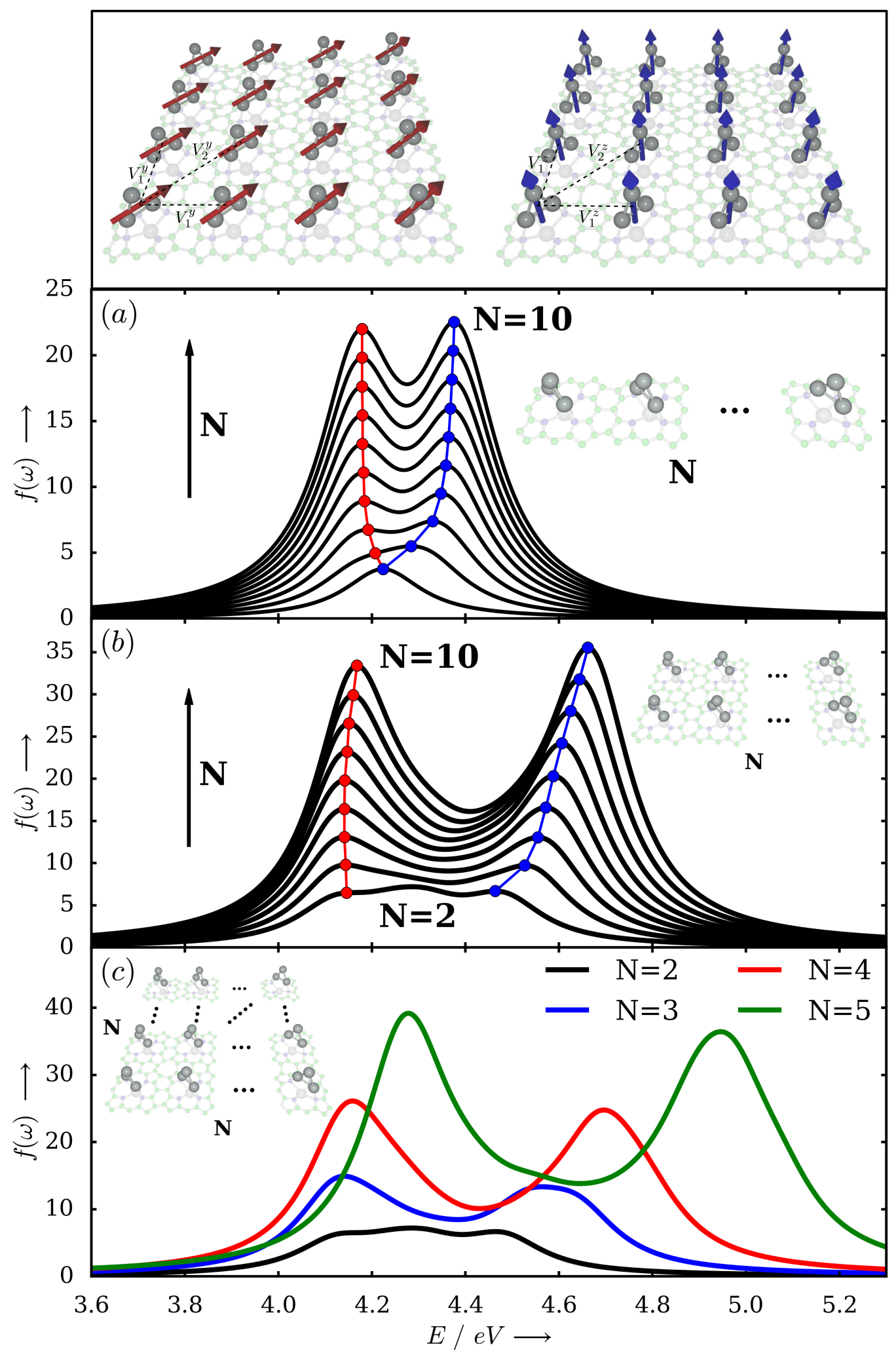

Figure 5: Top: Structures of Ag cluster arrays at 2D porphyrin template. Transition dipole moments corresponding to the discussed transitions are shown by arrows. a) Spectra of a linear system with increasing size, b) spectra of an 1D array consisting of two rows, c) spectra of square arrays with increasing size. 


\section{Extended planar structures}

The optical properties of extended planar $\mathrm{Ag}_{3}^{+}$arrays are studied employing the ab initio parametrized Frenkel exciton model where the coupling has been calculated using the transition density cube method (TDC). ${ }^{33}$ Although in this approach the porphyrin templates are neglected, the TDDFT calculations on the dimer and trimer show that this is justified since intracluster transitions are only slightly affected by the porphyrin surrounding. The structures of linear arrays with increasing size are shown in the inset of Fig. 5a). The corresponding absorption spectra presented in Fig. 5a) demonstrate the incremental splitting of the degenerate pair of excited states from $0.08 \mathrm{eV}$ in the dimer and $0.14 \mathrm{eV}$ in the trimer up to $0.2 \mathrm{eV}$ in a tape consisting of 10 subunits. The second class of systems we study consists of two cluster rows extending in one direction (see inset of Fig. 5b)). Here, a new type of coupling between the second-to-nearest neighbors along the diagonal occurs. A simple estimate based on the dipole-dipole approximation shows that the magnitude of the coupling has a value of $-0.71 \mu^{2} / \mathrm{R}^{3}$ in y-direction (J-coupling) and $0.35 \mu^{2} / \mathrm{R}^{3}$ (H-coupling) in z-direction. The presence of these additional couplings in a two-row system leads to higher excitonic splitting, which systematically increases with size (cf. Fig. 5b), reaching a value of 0.49 $\mathrm{eV}$ in a system with 20 cluster subunits. This effect is even larger in $2 \mathrm{D}$ quadratic arrays, as shown in Fig. 5c. For example, in a 5x5 array the excitonic splitting amounts to $0.77 \mathrm{eV}$. Altogether, these findings demonstrate that the optical properties of ordered arrays of Ag clusters can be systematically tuned by exploiting the excitonic couplings in nanoarchitectures with precisely controlled structural properties imposed by the porphyrin templates.

\section{Extended tilted structures}

Thorough investigation of the $\mathrm{Ag}_{3}^{+}$cluster orientation in the fully-optimized structures of the porphyrin- $\mathrm{Ag}_{4}$ dimer and trimer presented in Fig. 1 demonstrates that the relative shift and rotation of two neighboring clusters is preserved within both oligomers. Additional optimization of $2 \times 2$ and $3 \times 3$ porphyrin- $\mathrm{Ag}_{4}$ array structures (shown in Fig. 6) confirmed that not only the distance but also the rotation angles between neigboring $\mathrm{Ag}_{3}^{+}$clusters coincide with the ones determined 

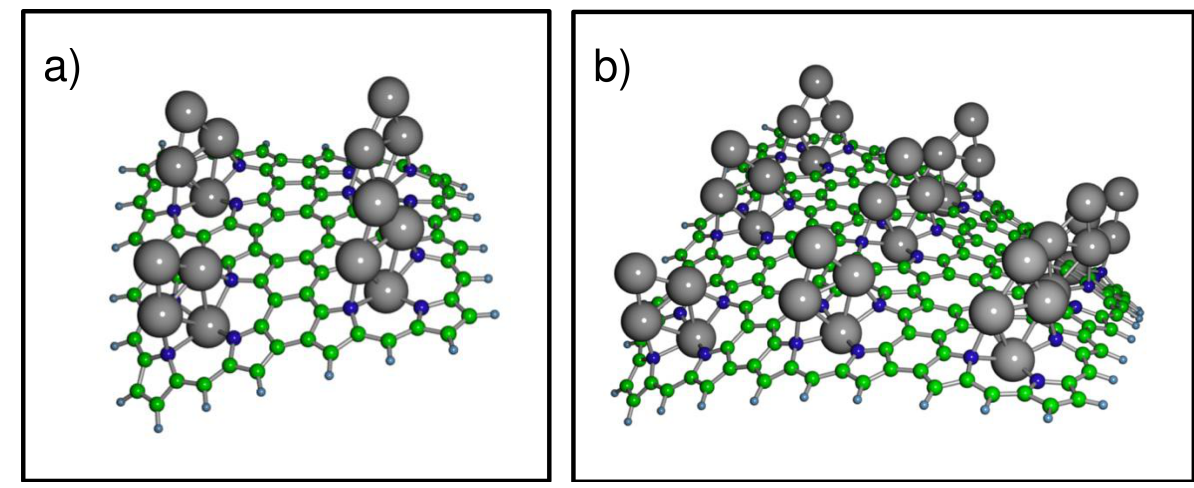

Figure 6: a) Optimized structure of a 2x2 silver-porphyrin array. b) Optimized structure of an 3X3 array. Both calculations have been carried out using the RI-DFT method.

based on the 1D structures. Therefore, these shift vector and rotation matrix can be considered as the characteristic properties of the porphyrin- $\mathrm{Ag}_{4}$ arrays and thus can be used to predict configurations of larger arrays.

Applying the least mean square fitting procedure to the fully-optimized $2 \times 2$ structure presented in Fig. 6a), the shift vectors $R_{h}, R_{v}$ and rotation matrices $\mathrm{M}_{h}, \mathrm{M}_{v}$ have been determined, which transform the coordinates of an $\mathrm{Ag}_{3}^{+}$cluster $r_{0}$ to the coordinates of its nearest neighbor along the vertical, $r_{v}$, or the horizontal, $r_{h}$, as follows:

$$
r_{v, h}=R_{v, h}+\mathrm{M}_{v, h} r_{0}
$$

Based on Eq. (22), an $\mathrm{Ag}_{3}^{+}$array of arbitrary size can be constructed with coordinates of each single cluster given by

$$
r_{i j}=\sum_{k=0}^{i=1} \mathbf{M}_{h}^{k} R_{h}+\sum_{k=0}^{j=1} \mathbf{M}_{v}^{k} \mathbf{M}_{h}^{i} R_{v}+\mathbf{M}_{v}^{j} \mathbf{M}_{h}^{i} r_{0}
$$

The correspondence between the $\mathrm{Ag}_{3}^{+}$arrays constructed by means of Eq. (23) and the fully optimized structures is reasonably good. Indeed, the root-mean-square deviations between the generated and optimized configurations of $\mathrm{Ag}_{3}^{+}$clusters are 0.04 and $0.29 \AA$ per cluster for dimer and trimer 1D structures (see Fig. 1) and 0.04 and $0.23 \AA$ per cluster for $2 \times 2$ and $3 \times 3$ arrays presented in Fig. 6. 
Using the methodology described above, the absorption spectra of $\mathrm{Ag}_{3}^{+}$arrays resembling such "free" porphyrin- $\mathrm{Ag}_{4}$ oligomers have been calculated. Relative tilt between the interacting clusters has been taken into account by applying the transformation (23) to the electronic and transition densities used in Eqs. (9) and (10) to calculate matrix interaction elements. The results are presented in Fig. 7

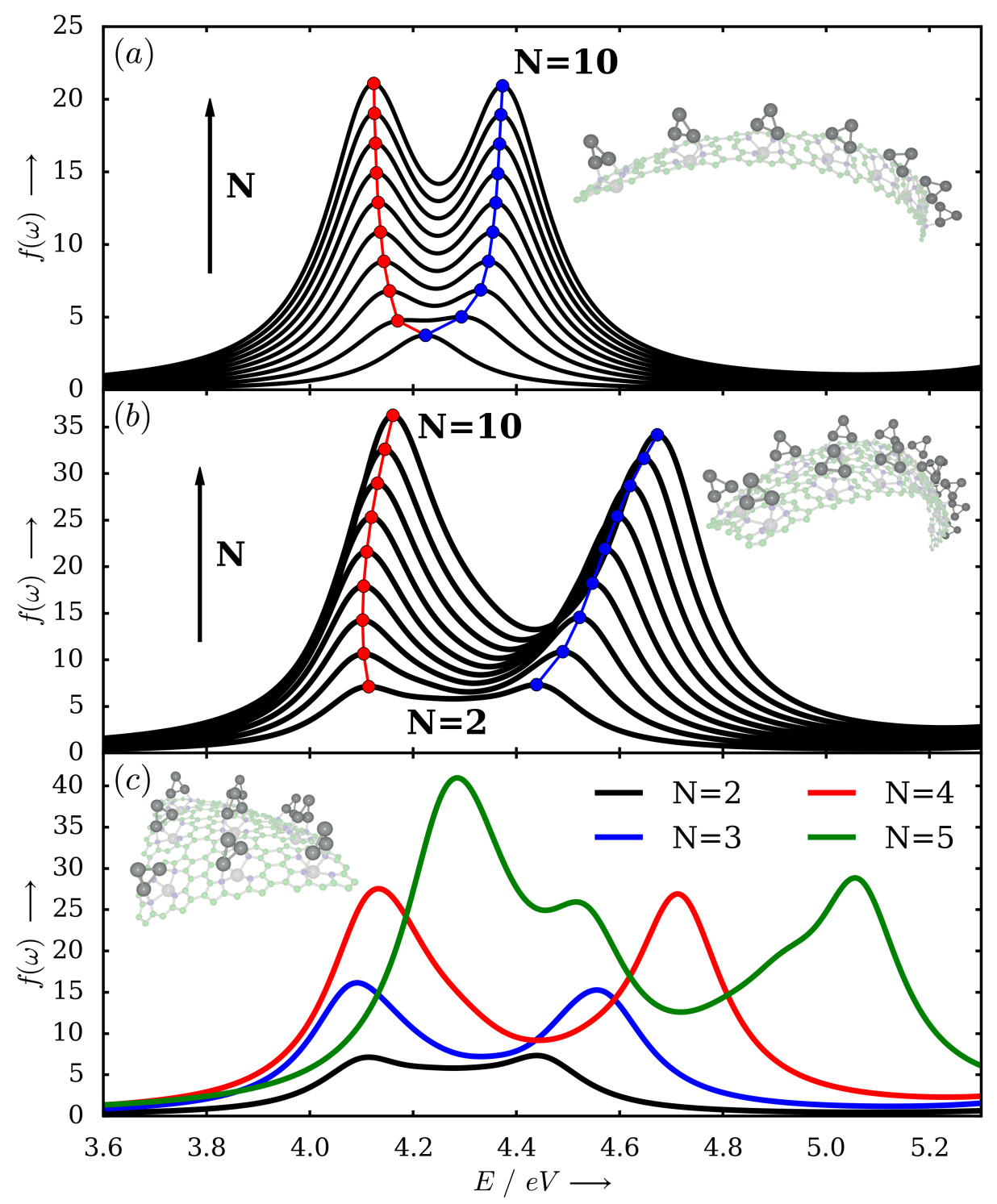

Figure 7: a) Spectra of a linear tilted system with increasing size, b) spectra of an 1D tilted array consisting of two rows, c) spectra of tilted square arrays with increasing size.

In part a) of Fig. 7, the absorption spectra of increasing tilted one-row tapes are presented, exhibiting an energetic splitting of $0.25 \mathrm{eV}$ in a system consisting of 10 subunits. In case of the 
aggregate comprising tilted two-cluster-rows with 20 subunits, the splitting amounts up to 0.51 $\mathrm{eV}$, as illustrated in part b) of Fig. 7. The less intense band present at $4.3 \mathrm{eV}$ in the absorption spectra of the flat two-row system cannot be observed here. In case of the quadratic, tilted arrays, the excitonic splitting amounts to $0.79 \mathrm{eV}$ in a $5 \times 5$ array. The differences in comparison with the energetic splitting in flat structures can be explained by looking at the magnitude and direction of the coupling again:

In tilted structures, coupling to the closest neighbor in y direction has a value of $-0.55 \mu^{2} / \mathrm{R}^{3}$ (in flat arrays $-0.5 \mu^{2} / \mathrm{R}^{3}$ ), and $0.84 \mu^{2} / \mathrm{R}^{3}$ in $\mathrm{z}$ direction (in flat arrays $1.0 \mu^{2} / \mathrm{R}^{3}$ ). Additionally, since $\mu_{y}$ and $\mu_{z}$ are not orthogonal in tilted arrays, the coupling between these transition dipole moments amounts to $-0.5 \mu^{2} / \mathrm{R}^{3}$ (in flat arrays it is zero). In tilted $2 \mathrm{D}$ structures, coupling between second-to-nearest neighbors along the diagonal has to be taken into account again and in case of the two-row system it results in $-0.47 \mu^{2} / \mathrm{R}^{3}$ in y direction (in flat arrays $-0.71 \mu^{2} / \mathrm{R}^{3}$ ), $0.18 \mu^{2} / \mathrm{R}^{3}$ in $\mathrm{z}$ direction (in flat arrays $0.35 \mu^{2} / \mathrm{R}^{3}$ ), and $-0.31 \mu^{2} / \mathrm{R}^{3}$ between $\mu_{y}$ and $\mu_{z}$ (in flat arrays 0 ). These mixed-direction splitting can be held responsible for the stronger shift in energy in comparison with the flat structures.

\section{Conclusion}

In conclusion, we have demonstrated that small silver clusters can be assembled in one and twodimensional arrays on porphyrin templates. Furthermore, by employing TDDFT calculations, we could show that the intrinsic optical properties of these clusters are largely preserved, but undergo $\mathrm{J}$ - and H-type excitonic coupling leading to energetic splitting. By employing the Frenkel exciton model we predict a splitting of up to $0.77 \mathrm{eV}$ in extended flat $2 \mathrm{D}$ arrays and $0.79 \mathrm{eV}$ in tilted arrays. These findings indicate that 2D cluster organic frameworks might open a new route towards the design of materials with tailored optical properties, which could serve as building blocks for plasmonically enhanced light-harvesting materials, sensors or cluster-mediated catalysts. 


\section{Supporting Information Available}

Absorption spectrum of the bare $\mathrm{Ag}_{3}^{+}$cluster, stable isomers of porphyrin- $\mathrm{Ag}_{4}$ monomer and dimer. This material is available free of charge via the Internet at http://pubs . acs .org/.

\section{Acknowledgement}

The funding by the ERC Consolidator Grant DYNAMO (Grant No. 646737) is gratefully acknowledged.

\section{References}

(1) Wasielewski, M. R. Self-Assembly Strategies for Integrating Light Harvesting and Charge Separation in Artificial Photosynthetic Systems. Acc. Chem. Res. 2009, 42, 1910-1921.

(2) Sengupta, S.; Würthner, F. Chlorophyll J-Aggregates: From Bioinspired Dye Stacks to Nanotubes, Liquid Crystals, and Biosupramolecular Electronics. Acc. Chem. Res. 2013, 46, $2498-2512$.

(3) Kasha, M.; Rawls, H. R.; El-Bayoumi, M. A. The exciton model in molecular spectroscopy. Pure Appl. Chem. 1965, 11, 371-392.

(4) May, V.; Kühn, O. Charge and Energy Transfer Dynamics in Molecular Systems, 3rd ed.; Wiley-VCH: Weinheim, Germany, 2011.

(5) Xia, Y.; Xiong, Y. J.; Lim, B.; Skrabalak, S. E. Shape-Controlled Synthesis of Metal Nanocrystals: Simple Chemistry Meets Complex Physics? Angew. Chem. Int. Ed. 2009, $48,60-103$.

(6) Mulvaney, P. Surface plasmon spectroscopy of nanosized metal particles. Langmuir 1996, $12,788-800$. 
(7) Harbich, W.; Fedrigo, S.; Buttet, J. The optical-absorption spectra of small silver clusters (N = 5-11) embedded in Argon matrices. J. Chem. Phys. Lett. 1992, 195, 613-617.

(8) Ramakrishna, G.; Varnavski, O.; Kim, J.; Lee, D.; Goodson, T. Quantum-sized gold clusters as efficient two-photon absorbers. J. Am. Chem. Soc. 2008, 130, 5032-5033.

(9) Lisinetskaya, P. G.; Mitric, R. Ab initio simulations of light propagation in silver cluster nanostructures. Phys. Rev. B 2014, 89, 035433.

(10) Lisinetskaya, P. G.; Mitric, R. Optimal control of light propagation and exciton transfer in arrays of molecular-like noble-metal clusters. Phys. Rev. B 2015, 91, 125436.

(11) Cuerva, M.; Garcia-Fandino, R.; Vazquez-Vazquez, C.; Lopez-Quintela, M. A.; Montenegro, J.; Granja, J. R. Self-Assembly of Silver Metal Clusters of Small Atomicity on Cyclic Peptide Nanotubes. ACS Nano 2015, 9, 10834-10843.

(12) Yu, J.; Choi, S.; Dickson, R. M. Shuttle-Based Fluorogenic Silver-Cluster Biolabels. Angew. Chem. Int. Ed. 2009, 48, 318-.

(13) Richards, C. I.; Choi, S.; Hsiang, J. C.; Antoku, Y.; Vosch, T.; Bongiorno, A.; Tzeng, Y. L.; Dickson, R. M. Oligonucleotide-stabilized Ag nanocluster fluorophores. J. Am. Chem. Soc. 2008, 130, 5038-5039.

(14) O’Neill, P. R.; Young, K.; Schiffels, D.; Fygenson, D. K. Few-Atom Fluorescent Silver Clusters Assemble at Programmed Sites on DNA Nanotubes. Nano Lett. 2012, 5464-5469.

(15) Copp, S. M.; Schultz, D. E.; Swasey, S.; Gwinn, E. G. Atomically Precise Arrays of Fluorescent Silver Clusters: A Modular Approach for Metal Cluster Photonics on DNA Nanostructures. ACS Nano 2015, 9, 2303-2310.

(16) Kulesza, A.; Mitric, R.; Bonacic-Koutecky, V.; Bellina, B.; Compagnon, I.; Broyer, M.; Dugourd, R. A. P. Doubly Charged Silver Clusters Stabilized by Tryptophan: Ag-4(2+) as 
an Optical Marker for Monitoring Particle Growth. Angew. Chem. Int. Ed. 2011, 50, 878881.

(17) Kumar, S.; Bolan, M. D.; Bigioni, T. P. Glutathione-Stabilized Magic-Number Silver Cluster Compounds. J. Am. Chem. Soc. 2010, 132, 13141-13143.

(18) Tabarin, T.; Kulesza, A.; Antoine, R.; Mitric, R.; Broyer, M.; Dugourd, P.; BonacicKoutecky, V. Absorption Enhancement and Conformational Control of Peptides by Small Silver Clusters. Phys. Rev. Lett. 2008, 101, 213001.

(19) Bebensee, F.; Svane, K.; Bombis, C.; Masini, F.; Klyatskaya, S.; Besenbacher, F.; Ruben, M.; Hammer, B.; Linderoth, T. R. A Surface Coordination Network Based on Copper Adatom Trimers. Angew. Chem. Int. Ed. 2014, 53, 12955-12959.

(20) Hu, Q.; Jin, D.; Nam, S. H.; Xiao, J.; Liu, Y.; Zhang, X.; Fang, N. X. Ultrafast fluorescent decay induced by metal-mediated dipole-dipole interaction in two-dimensional molecular aggregates. 2016,

(21) Auwärter, W.; Ecija, D.; Klappenberger, F.; Barth, J. V. Porphyrins at interfaces. Nature Chem. 2015, 7, 105-120.

(22) Cho, H.; Jeong, D. H.; Cho, S.; Kim, D.; Matsuzaki, Y.; Tanaka, K.; Tsuda, A.; Osuka, A. Photophysical properties of porphyrin tapes. J. Am. Chem. Soc. 2002, 124, 14642-14654.

(23) Nakamura, Y.; Aratani, N.; Shinokubo, H.; Takagi, A.; Kawai, T.; Matsumoto, T.; Yoon, Z. S.; Kim, D. Y.; Ahn, T. K.; Kim, D. et al. A directly fused tetrameric porphyrin sheet and its anomalous electronic properties that arise from the planar cyclooctatetraene core. J. Am. Chem. Soc. 2006, 128, 4119-4127.

(24) Wiengarten, A.; Seufert, K.; Auwärter, W.; Ecija, D.; Diller, K.; Allegretti, F.; Bischoff, F.; Fischer, S.; Duncan, D. A.; Papageorgiou, A. C. et al. Surface-assisted Dehydrogenative Homocoupling of Porphine Molecules. J. Am. Chem. Soc. 2014, 136, 9346-9354. 
(25) Lu, Y. Z.; Chen, W. Sub-nanometre sized metal clusters: from synthetic challenges to the unique property discoveries. Chem. Soc. Rev. 2012, 41, 3594-3623.

(26) Attia, Y. A.; Buceta, D.; Blanco-Varela, C.; Mohamed, M. B.; Barone, G.; LopezQuintela, M. A. Structure-Directing and High-Efficiency Photocatalytic Hydrogen Production by Ag Clusters. J. Am. Chem. Soc. 2014, 136, 1182-1185.

(27) von Arnim, M.; Ahlrichs, R. Performance of parallel TURBOMOLE for density functional calculations. J. Comp. Chem. 1998, 19, 1746-1757.

(28) Lee, C. T.; Yang, W. T.; Parr, R. G. Development of the Colle-Salvetti correlation-energy formula into a functional of the electron-density. Phys. Rev. B 1988, 37, 785-789.

(29) Yanai, T.; Tew, D. P.; Handy, N. C. A new hybrid exchange-correlation functional using the Coulomb-attenuating method (CAM-B3LYP). Chem. Phys. Lett. 2004, 393, 51-57.

(30) Andrae, D.; Haeussermann, U.; Dolg, M.; Stoll, H.; Preuss, H. Energy-adjusted abinitio pseudopotentials for the 2nd and 3rd row transition-elements. Theor. Chim. Acta 1990, 77, $123-141$.

(31) Weigend, F.; Ahlrichs, R. Balanced basis sets of split valence, triple zeta valence and quadruple zeta valence quality for $\mathrm{H}$ to Rn: Design and assessment of accuracy. Phys. Chem. Chem. Phys. 2005, 7, 3297-3305.

(32) Bonacic-Koutecky, V.; Pittner, J.; Boiron, M.; Fantucci, P. An accurate relativistic effective core potential for excited states of Ag atom: An application for studying the absorption spectra of Ag-n and Ag-n(+) clusters. J. Chem. Phys. 1999, 110, 3876-3886.

(33) Krueger, B. P.; Scholes, G. D.; Fleming, G. R. Calculation of couplings and energy-transfer pathways between the pigments of LH2 by the ab initio transition density cube method. $J$. Phys. Chem. B 1998, 102, 5378-5386. 
(34) Werner, U.; Mitrić, R.; Bonačić-Koutecký, V. Simulation of time resolved photoelectron spectra with Stieltjes imaging illustrated on ultrafast internal conversion in pyrazine. J. Chem. Phys. 2010, 132, 174301.

(35) Casida, M. E. In Recent Advances in Density Functional Methods; Chong, D. P., Ed.; World Scientific: Singapore.

(36) Dreuw, A.; Head-Gordon, M. Single-reference ab initio methods for the calculation of excited states of large molecules. Chem. Rev. 2005, 105, 4009-4037.

(37) Christiansen, O.; Koch, H.; Jorgensen, P. The 2nd-order aproximate coupled-cluster singles and doubles model CC2. Chem. Phys. Lett. 1995, 243, 409-418.

(38) Dorough, G. D.; Miller, J. R.; Huennekens, F. M. Spectra of the metallo-derivatives of alpha,beta,gamma,omega-Tetraphenylporphine. J. Am. Chem. Soc. 1951, 73, 4315-4320.

(39) Compagnon, I.; Tabarin, T.; Antoine, R.; Broyer, M.; Dugourd, P.; Mitric, R.; Petersen, J.; Bonacic-Koutecky, V. Spectroscopy of isolated, mass-selected tryptophan-Ag-3 complexes: A model for photoabsorption enhancement in nanoparticle-biomolecule hybrid systems. $J$. Chem. Phys. 2006, 125, 164326-1-164326-5.

(40) Gouterman, M. Spectra of Porphyrins. J. Mol. Spec. 1961, 6, 138-163.

(41) Yamaguchi, Y. Time-dependent density functional calculations of fully pi-conjugated zinc oligoporphyrins. J. Chem. Phys. 2002, 117, 9688-9694. 